par ce que nous avait donné une recherche analogue, pour laquelle nous avions utilisé le phosphate monocalcique. Nous avions vu qu'un caséinate à 630 , de $p H=7,25$, coagule par le lab avec une quantité de phosphate monocalcique d'environ 0,500 gr. par litre; or à cette quantité ne répond que $0,115 \mathrm{gr}$. de chaux. La quantité de $\mathrm{CaCl}^{2}$ qui renferme le même quantum de chaux est le double : $0,230 \mathrm{gr}$.

Il semble done qu'il faille moins de chaux sous la forme de phosphate monocalcique que sous la forme de chlorure de calcium pour provoquer la coagulation d'un même caséinate sous l'action du lab, mais nous devons faire remarquer que la différence, au total, n'est pas considérable, puisqu'elle se chiffre par 0,050 gr. par litre, même pas $7 \%$ de la chaux totale du caséinate et du chlorure. Nous ajouterons que la réaction finale est beaucoup plus acide avec le phosphate qu'avec le chlorure de calcium. Le $p H$, en effet, est de 6,85 avec l'association caséinate de calcium + phosphate monocalcique; il est de 7,06 avec l'association caséinate de calcium + chlorure de calcium. Si nous acidifions cette dernière avec beaucoup de précaution, cela va sans dire, pour amener son $p H$ à 6,85 , la coagulation se fera rapidement, et en quelques secondes, nous obtenons un bloc ferme. L'acide ajouté a décalcifié partiellement le caséinate à 630 , comme l'a fait d'ailleurs le phosphate monocalcique, et quand nous arriverons au même $p H$, nous aurons en présence des systèmes extrêmement voisins. On comprend done qu'ils coagulent par la présure dans des conditions presque identiques.

(A suivre.)

\title{
LES MALPROPRETÉS VISIBLES DU LAIT LEUR EXAMEN MICROSCOPIQUE
}

\author{
par R. LOTH,
}

Docteur - Vétérinaire

(Fin)

\section{Cellulies épidermiques - Cellules épithéltales}

Origine. - On rencontre d'une façon à peu près constante dans les laits des cellules épidermiques, voire même des lambeaux épidermiques. Leur présence si fréquente s'explique facilement. Ces cellules appartiennent aux couches supérieures de l'épithélium lamellaire de la peau extérieure du trayon, et aussi de la peau des mains du trayeur. Dans le travail mécanique intensif auquel sont soumises les tétines du fait de l'excitation de celles-ci par la traite, excitation qui porte sur une partie de la glande en érection, les couches superficielles de la peau de la mamelle sont soumises continuellement à des frottements multiples et leur exfoliation s'ensuit naturellement. Il est facile d'ailleurs de se rendre compte de ce phénomène : si nous regardons une mamelle avant 
la traite, les trayons sont ridés, et, si l'on passe doucement la main à leur surface, on sent la peau rugueuse, nous dirons même, râpeuse. Si nous recommençons après la traite, la peau est devenue souple, lisse, parce que les couches superficielles de l'épiderme, notamment la couche cornée qui donnait aux trayons cette sensation de rugosité, ont disparu. Le même phénomène se note pour les mains du trayeur.

Description. - Au faible grossissement (fig. 6), on aperçoit des petites masses jaunâtres ou rose très pâle qui paraissent d'une certaine épaisseur, puisqu'en faisant varier la vis micrométrique, divers plans en profondeur sont perçus.

$\mathrm{Au}$ fort grossissement (fig. 7), les taches présentent des divisions longitudinales formées par des sillons sinueux dont on aperçoit les reliefs latéraux. Parfois, certaines de ces exfoliations présentent encore des divisions qui sont orientées dans tous les sens, formant une véritable mosaïque et faisant prendre à l'impureté un aspect écailleux.

On trouve aussi des cellules provenant de l'épithélium du canal du trayon et des canaux lactifères ; ce sont alors des lamelles fines aux contours arrondis, ovales ou sinueux, apparaissant souvent repliées sans structure particulière, des cellules isolées ou réunies qui proviennent du stratum mortificateur de l'épithélium lamellaire. Si on ne décèle aucune structure particulière, on peut cependant parfaitement reconnaître parmi elles des noyaux ronds et plats.

\section{Débris ALIMENTAIRES}

Origine. - Ces impuretés sont constituées par des débris provenant de l'alimentation ordinaire des animaux, c'est-à-dire : foin, luzerne ou trèfle, paille, balles d'avoine, son, etc...

Généralement, la traite s'effectue au moment des repas des animaux ; ceux-ci, en effet, étant occupés, sont plus calmes et s'agitent moins pendant la mulsion. Les animaux tirent alors le fourrage du ratelier par des mouvements souvent brusques, ce qui fait que des poussières faites de débris fourragers plus ou moins menus se dégagent et viennent tomber en partie dans le seau du trayeur.

On peut admettre encore que le lait fraîchement trait et abandonné dans son seau non recouvert est exposé à toutes les poussières fourragères de l'étable.

Description. - Une description détaillée serait inutile, et il suffit d'ouvrir un ouvrage de botanique pour y trouver les figures microscopiques que nous rencontrons dans le lait à ce point de vue. Les grosses impuretés nagent à la surface du liquide sous forme de brins, de feuilles, de parcelles verdâtres ou jaunâtres. Le faible grossissement suffit à reconnaître la nature des impuretés plus petites. Sous le microscope, 
on aperçoit des parcelles verdâtres ou jaunâtres irrégulières, des filaments ligneux, des faisceaux assemblés, etc..., etc...

Matì̀res minérales, RoUILle, ALUMine, ETC. (fig. 6, 7 et 10)

Origine. - Los particules d'hydrate de fer ou d'oxyde de fer que l'on rencontre dans le lait sont très nombreuses. Les fragments microscopiques de rouille proviennent des récipients dans lesquels le lait est déversé et manipulé. Ces récipients, en général, sont étamés, mais trop souvent, une fois que l'étain est parti, on tarde à les faire réparer. Aussi n'est-il pas rare de rencontrer dans les exploitations de la campagne des pots ou des seaux à lait dont la plus grande partie des parois intérieures sont rouillées.

Description. - Ces particules se reconnaissent à leur forme : petites plaquettes polyédriques, assez irrégulières, à angles plus ou moins arrondis ; leur couleur est jaunâtre, jaune brunâtre ; parfois ce sont même de simples petites taches grisâtres, gris-brun.

Si l'examen microscopique ne suffit pas à les identifier, on peut facilement mettre en évidence leur origine. On ajoute entre lame et lamelle une trace de sulfocyanure de potassium et d'acide chlorhydrique, et l'on voit presque immédiatement les particules se colorer en rose.

On rencontre également des particules de plomb, d'alumine, et surtout de ces dernières, car, de plus en plus, on utilise des bidons en aluminium. Los premières sont un peu plus foncées que les secondes. Une goutte d'iodure de potassium colore en jaune les particules de plomb lorsqu'on l'étale entre lame et lamelle.

\section{DÉBRIS DE CHARBON (fig. 5 et 11)}

Origine. - Nous les rencontrons souvent en hiver, où elles sont excessivement abondantes. Les bidons de lait ou tous autres récipients de la laiterie sont exposés dans les salles, dans les magasins où l'on fait du feu, et la poussière de charbon vient s'y déposer. On rencontre également cette poussière dans le lait transporté en chemin de fer, car certains bidons sont mal bouchés et les poussières menues de la fumée de la locomotive peuvent y pénétrer.

$\mathrm{Au}$ microseope, on voit disséminées dans le champ des masses irrégulières d'un noir franc.

\section{INSECTES ET PARASITES}

La provenance des insectes et des parasites est de deux ordres : il en est, et ce sont les plus fréquents, qui viennent du milieu extérieur : mouches, insectes très variés qui viennent se nourrir de lait et qui y tombent le plus souvent. Les autres proviennent de l'animal luimême. Nous reconnaîtrons d'abord les ectoparasites, et nous y rangerons 
les poux, les lentes collées aux poils. Dans un lait provenant de vaches qui étaient en traitement à l'Ecole Vétérinaire, nous avons rencontré un acarien. Au fort grossissement (560 diamètres), ilavaitles caractéristiques d'un psorote ou d'un choriopte, mais il était beaucoup plus petit ; c'était un tyroglyphe de la famille des sarcoptidés, simple saprozoïte.

Nous trouvons dans le lait également des parasites provenant de l'appareil digestif de la vache, mais plus souvent l'œuf ou la lente que l'insecte complet que nous rencontrons. C'est ainsi que les oeufs de strongles, de douves surtout sont une trouvaille habituelle. Ces coufs sont amenés dans le lait avec les matières excrémentitielles, et, du fait de la fréquence de ces impuretés, on en peut conclure à la présence fréquente également de ces parasites chez les animaux qui fournissent le lait à examiner.

\section{QUELQUES DOSAGES DES IMPURETÉS}

L'étude quantitative des impuretés du lait n'est pas précisément un des buts de cette étude, car nous avons préféré nous limiter à l'étude qualitative, mais il nous semble qu'en donnant certains résultats pondéraux, nous ajouterons quelque intérêt aux données antérieurement fournies. Les dosages ont été faits en employant une des méthodes indiquées au début de cette étude, mais de préférence à celle qui utilise la centrifugation.

Nous nous sommes assuré que nous ne pesions vraiment que les impuretés, en procédant à un examen microscopique qui nous a montré que ces dernières étaient tout à fait dégagées d'éléments propres du lait, tels que la matière grasse et les dépôts caséineux.

10 L'échantillon a été recueilli dans un bidon provenant d'un centre de ramassage, et examiné avant le tamisage du lait :

Impuretés restées dans le lait ............ 0 gr. 047 par li1 re

Impuretés restées dans la crème ............ 0 gr. 015 ,

Impuretés totales ................... 0 gr. 062 ,

$2^{\circ}$ Dosage opéré dans les mêmes conditions, mais sur un lait provenant d'un autre centre de ramassage :

Impuretés du lait restées dans le lait ......... 0 gr. 035 parditre

Impuretés de la crème $\ldots \ldots \ldots \ldots \ldots \ldots \ldots \ldots \ldots, 0$ gr. 012 ,

Impuretés totales .................... 0 gr. 047 "

$3^{\circ}$ Dosage sur un lait recueilli dans une cuve après avoir été filtré et pasteurisé :

Impuretés du lait . . . . . . . . .

Impuretés de la crème ................ 0 gr. 002 ,

Impuretés totales $\ldots \ldots \ldots \ldots \ldots \ldots \ldots \ldots \ldots, 0$ gr. 030 ,

$4^{\circ}$ Dosage sur un lait recueilli dans une euve après tamisage :

Impuretés totales . . . . . . . . . . 028 par litre 
Si l'on confronte ces divers résultats, on remarque d'abord qu'un tamisage, même bien fait, ne suffit pas à éliminer les impuretés du lait, mais qu'une très grande partie est retenue. On notera également, en comparant seulement les impuretés restant dans la crème, que le tamisage retient surtout les impuretés d'origine végétale, puisque ce sont elles qui "montent " avec la crème. Après filtration, le dosage montre une quantité minime d'impuretés dans la crème.

5o Dosage dans un lait acheté dans une boutique de quartier :

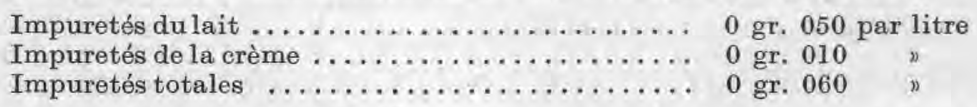

$6^{\circ}$ Dosage sur un lait acheté dans les mêmes conditions, mais dans une autre boutique :

Impuretés totales. . . . . . 035 par litre

$7^{\circ}$ Dosage sur un lait d'une troisième boutique :

Impuretés totales $\ldots \ldots \ldots \ldots \ldots \ldots \ldots \ldots \ldots, 0 \ldots \ldots$ gr. 050 par litre

Les chiffres trouvés à Christiania, à Berlin, représenteraient, d'après les auteurs qui les ont fournis, uniquement la quantité d'excréments trouvée dans le lait. Nous nous permettons de faire remarquer qu'il nous paraît bien difficile de séparer en totalité et uniquement les matières excrémentitielles, alors que les chiftres de nos dosages représentent toutes les impuretés. Nos chiffres n'ont, au surplus, rien qui doive surprendre, car est-il besoin de redire que les laits courants renferment d'une façon apparente des impuretés en grande quantité, et parmi celles-ci s'en trouvent qui sont assez denses, au point de se déposer très rapidement au fond du récipient. Nous estimons qu'elles ne sont pas toujours pesées comme elles le devraient.

\section{CONSIDÉRATIONS SUR LA PRODUCTION HYGIÉNIQUE DU LAIT ET SON CONTROLE}

Les pages qui précèdent nous ont montré que le lait ordinaire, le lait courant, est trop souvent un lait souillé de malpropretés fines, visibles évidemment, mais dont l'examen doit être fait au microscope. Le dépôt de ce lait est eonstitué par un piqueté gris noirâtre dans lequel on trouve tout ce que nous avons décrit dans les pages précédentes. Ces saletés visibles s'accompagnent toujours d'un ensemencement microbien, car elles servent de support à une très grande variété d'agents infiniment petits ; aussi, le lait malpropre visiblement est-il ipso facto un lait fortement ensemencé. On ne sait pas toujours quels sont les germes qu'il recèle; aussi ne faut-il pas repousser un lait malpropre simplement parce qu'il est peu appétissant, sinon même parfois répugnant, mais parce que derrière les saletés visibles se cache une flore 
microbienne qui peut être novice, dangereuse. La santé publique est en jeu ici, et il importe de la protéger.

Ainsi que le disait le Professeur Ch. Porcher, dans un rapport qu'il fit au Congrès International Vétérinaire de Londres, en 1914, lequel congrès fut arrêté par la déclaration de guerre : "Les hécatombes d'enfants qui surviennent aux temps chauds, les épidémies de fièvre typhoïde d'origine lactée, beaucoup plus nombreuses et meurtrières qu'on le croit, sont en relation étroite avec une large pollution du lait au moment de la traite. Les statistiques de tous les pays montrent que si la diarrhée infantile décime les enfants au-dessous de deux ans, c'est le lait sale qu'il faut partout considérer comme la cause primordiale de cette mortalité. " Il ne faut cesser de répéter que les ravages que font les affections gastro-intestinales dans la première enfance trouvent une cause importante dans le lait sale.

Nous n'avons pas l'intention ici de passer en revue la liste des microbes dangereux que peut nous apporter le lait. Nous ne ferons qu'en citer quelques-uns dont la présence est liée intimement à celle des diverses souillures que nous avons examinées antérieurement.

Ce sont les matières fécales qui sont, peut-on dire, les plus grands pourvoyeurs en microbes nuisibles. Ce sont elles qui apportent dans le lait le $B$. coli, le paratyphique $B$, le $B$. mesentericus, le $B$. proteus, le $B$. subtilis. Les mamelles, en dépit des précautions prises, sont toujours souillées par ces germes, lorsque les vaches couchent directement sur la litière, qui n'est pas toujours changée comme elle le devrait.

La première conclusion qui se dégage de cette constatation, c'est qu'il semble facile d'éliminer la plus grande partie de cette flore, sinon toute, en prenant les précautions de propreté visant surtout le nettoyage du pis. Ayons le courage de le reconnaître et de l'avouer, rien n'est fait le plus généralement.

Ceux qui connaissent le lait, ou qui devraient le connaitre par métier, par fonction, parce qu'ils en vivent, ignorent tout de sa sensibilité et se figurent qu'il n'y a rien à faire pour le protéger. Ils courbent la tête devant l'inévitable qu'ils pourraient éviter.

Producteurs et consommateurs, les deux extrémités de la chaîne, ont besoin d'une éducation sérieuse. Parlant du consommateur, M. RENNES dit : "Si les étables sont sales, les pièces où l'on tient le lait sontelles toujours très propres? Si les bidons, les pots des laitiers demeurent à découvert, exposés aux souillures, ne voit-on jamais de casseroles, de pots, de bols attendre aux seuils des portes, à découvert aussi, en compagnie du pain et sur le paillasson ? En admettant que le lait arrive propre et sain chez le consommateur, il est peu de maisons où l'on sache l'entourer de soins nécessaires pour qu'il se conserve tel. »

En ce qui concerne la production, depuis des années, on semble se préoccuper avec juste raison des conditions d'hygiène dont elle doit 
être entourée. Et le rôle, l'activité de notre Maître, qui fut dans ces questions un animateur remarquable, n'ont plus besoin d'être rappelés. Nous n'a vons pas ici la prétention d'éla borer les grandes règles destinées à régir la production d'un lait propre ; elles sont connues de tous, et dans bəaucoup de pays les efforts faits dépassent de beaucoup ceux qui ont été jusqu'ici tentés en France. Mais nous voudrions faire quelques remarques pouvant s'appliquer à l'obtention d'un pareil lait. Il est bien de prôner la construction d'étables modèles, mais le temps est loin qui nous pormettra de constater partout que ces établissements ne prêtent pas le flane à la moindre critique. D'ailleurs est-il si nécessaire que cela d'avoir des établissements luxueux, construits à grand renfort d'argent? Los recherches si intéressantes faites à l'Institut de Laiterie de Rsading, sous la conduite de M. le Professeur Stenhouse Wirtiams, ont montré que même dans des étables qui sont pour les vaches ce que le taudis est malheureusement trop souvent pour l'homme, on peut obtenir du lait propre si le vacher sait prendre les précautions qu'il faut. Ces précautions, e'est la propreté de l'animal, de la litière, et celle du milieu, malgré la misère apparente qu'il affecte. C'est la propreté du pis, du train de derrière, celle de la litière et des seaux, c'est l'abus de l'eau chaude et de l'eau froide pour supprimer toutes les poussières, pour les condenser en quelque sorte. Panser l'animal, éviter la formation d'une véritable carapace de bouse desséchée, dont sont enduites ses cuisses, tout son arrière-train, voilà quelque chose qui est à la portée de tous. Il n'y" a aueun luxe dans tous ces soins : laver la mamelle avant Ia traite, la sécher, donner des habits propres au vacher, lui faire laver les mains, mettre à sa disposition des récipients propres et sess, c'est la propreté pure et simple, mais une propreté vraie qui est pleine d'heureux résultats. Malheureusement, en dépit de leur simplicité, ces prescriptions sont à l'heure actuelle le plus souvent lettre morte, paree que d'abord tout ce qui concerne l'hygiène semble en quelque sorte exclu des étables, ensuite, parce que l'immense majorité des produsteurs est ignorante de la signification des soins de propreté qu'on lui demande. Ce qu'il y a de plus lamentable, c'est que non seulement ils ignorent tout cela, mais qu'ils veulent peut-être les ignorer, tellement ces précautions leur semblent superflues.

Il y a donc là une mentalité à réformer, et comme il ne faut pas toujours attendre un résultat tangible d'une discipline qui vienne du producteur lui-même, on se demande dans quelle mesure l'obligation et la contrainte peuvent jouer dans l'organisation du contrôle de la production du lait.

Ce contrôle existe en théorie, puisqu'il est prévu par un décret du 25 mars 1924 ; en fait, jusqu'ici, il est pour ainsi dire inexistant, parce - qu'on a reculé devant les réolamations, d'aucuns diraient les criailleries des producteurs qui n'ont pas toujours compris de quel côté était leur 
véritable intérêt. S'il faut que le contrôle s'abrite derrière des pouvoirs de police qu'on est toujours tenté d'exagérer, il n'arrivera à rien. Contrôle ici veut dire collaboration du contrôlé et du contrôleur, et il ne faudra sévir que lorsque la mauvaise volonté aura été évidente et continuelle. Evidemment, la tâche est immense, mais ce n'est pas une raison pour ne pas l'entreprendre, et encore une fois, des efforts intéressants, qui ont donné des résultats dignes d'être remarqués, sont faits ailleurs. Pourquoi ne le seraient-ils pas en France?

Le consommateur doit payer à sa valeur un lait digne de ee nom, mais au sacrifice péeuniaire qu'il consent, le producteur doit répondre par un effort proportionné. La qualité du lait doit être garantie, et toute garantie pour être valable implique un contrôle effectif.

Il est triste de remarquer que toutes les impuretés que nous nous sommes employé à signaler dans le lait et dont nous avons rapidement indiqué le dosage font un total impressionnant quand on considère le nombre de kilogrammes consommés par une population d'une ville importante. On peut dire que certaines grandes villes consomment chaque année plusieurs tonnes d'excréments de vaches. Cela est-ce permis ?

$\mathrm{Au}$ surplus, nos législateurs timides n'ont rien innové. Depuis les temps les plus reculés, la question du lait n'a cessé de dominer le problème de l'alimentation des enfants. A Rome, elle a fait l'objet de mesures spéciales et nombreuses. En France, au xII' ${ }^{e}$ siècle, le Roi prend un arrêté réglementant le placement des enfants en nourrice. Louis XIV prend des mesures pour éviter l'écrémage et le mouillage et la souillure du lait. Madame de Genlis, dans les "Veillées du Château ", s'appesantit non seulement sur les avantages du lait pris à la ferme directement, mais sur l'importance d'une étable bien tenue.

Jusqu'à ces dernières années, il n'existait pas de lois ou de réglementation très étudiées permettant d'éliminer des laits malpropres. C'est le décret du 25 mars 1924 qui vient combler cette lacune par son article 2 :

( Ne peut être considéré comme lait propre à la consommation :

$1^{0}$ Le lait provenant d'animaux atteints de maladies dont la nomenclature sera donnée par arrêté du Ministre de l'Agriculture ;

$2^{\circ}$ Le lait coloré, malpropre ou malodorant;

3o Le lait provenant d'une traite opérée moins de sept jours après le part et d'une manière générale le lait contenant du colostrum. »

Ce décret sous-entend donc que le contrôle du lait vendu dans le commerce aura également une base hygiénique.

Il sera très difficile de déterminer d'une façon officielle ce qu'on entend par un lait malpropre et malsain. Faudra-t-il donner des chiffres ? Apprécier la malpropreté aux points ? On voit tout de suite, et ce que nous avons dit plus haut le montre bien, ce qu'a d'illusoire une pareille manière de faire. La filtration du lait sur des 
plaques de ouate nous permet bien de distinguer les laits en : lait très sale, lait sale, lait assez propre, lait propre, mais y a-t-il toujours proportionnalité entre la saleté visible et la saleté invisible plus dangereuse, c'est-à-dire la flore microbienne ? Ici, disons-le nettement, ce n'est pas cette méthode qui pourra donner de hons résultats, e'est la méthode préventive, celle qui eonsistera à faire campagne dans tout le pays, près des producteurs, pour les amener à leur montrer que leur véritable intérêt est de donner du lait propre, et non les laits trop souvent sales qu'ils jettent délibérément dans le commerce, car le lait ne vaut pas seulement par sa valeur chimique, mais aussi considéré du côté microbien. C'est aux syndicats de producteurs, à leurs dirigeants, d'exercer sur eux une pression éducative, à laquelle vraiment, avec l'aide du temps, ils ne pourront échapper.

Il devrait être interdit de vendre pour du bon lait un lait quelconque, dont on ne justifie pas la qualité par des soins qu'un contrôle bienveillant, mais sérieux, peut définir aisément. Rien dans tout cela ne va à l'encontre de la liberté du commerce. Un contrôle peut fonctionner sans gêner les commerçants, et enfin, la liberté du commerce n'est pas celle de vendre une marchandise suspecte, voire même dangereuse, une marchandise qui, en tout cas, vendue à l'industriel, ne lui permet pas d'en tirer tous les rendements qu'il en peut espérer.

" La liberté du commerce, dit le Professeur Ch. Porcher, a comme limite la défense de la santé publique. La plupart des pays autres que la France ont compris la question sous ce jour. "

Lə règlement du 25 mars 1924 nous dit également que l'on considérera comme impropre à la consommation des laits d'animaux atteints de maladies dont la nomenclature sera arrêtée par le Ministre de l'Agriculture. L'Administration, en s'engageant dans cette voie, n'a pas vu les difficultés qui vont se poser devant elle. Evidemment, ce texte devra être rédigé, mais l'on n'a pas encore vu sortir le décret ou l'arrêté ministériel qui stipule les maladies évoquées plus haut. Il ne sera pas facile à mettre sur pied. Une police sanitaire est donc à élaborer qui permettra de rendre vraiment opérant le texte naguère publié.

Enfin, reconnaissons que l'éducation du consommateur doit être faite en même temps que celle du producteur. Une propagande intelligente, appelant l'attention du consommateur sur la grande valeur du lait et des produits laitiers, est à entreprendre sans discontinuité. Il ne faut pas, pour se servir d'une expression de la guerre : " bourrer le crâne " au consommateur, lui dire que le lait ne coûte pas cher à produire et, par conséquent, qu'il ne doit pas être vendu cher. Il faut au contraire lui dire la vérité, toute la vérité, lui montrer que les efforts que l'on peut tenter pour améliorer la qualité du lait doivent être reconnus par lui-même, par son consentement à un prix d'achat plus élevé. C'est une erreur de dire qu'il n'y a qu'un lait, comme cela en 
est une aussi de dire qu'il est une infinité de laits, car une classification dont on multiplie les paliers ne peut qu'engendrer la confusion et, par suite, la fraude. Du jour où le consommateur aura compris ce que c'est que le lait au point de vue alimentaire, tout ce qu'on en peut tirer de riche et de précieux, se sera rendu nettement compte qu'il ne coûte pas rien à produire, sans doute sera-t-il disposé à payer plus cher tout lait qui possédera, un contrôle intelligent et bien organisé le lui aura dit, - une valeur hygiénique supérieure.

\section{BIBLIOGRAPHIE}

(1) Wilhelm ERNst. - Milchygiene für Tierärate, $2^{e}$ Edition. - Ferdinand Enke, Editeur, Stuttgart.

(2) SEIFFert. - Über Milchschmütz und seine Bekämpfung. - Fortschritte der Medizin, 1908.

(3) C. Ró́cand. - Faut-il faire bouillir le lait? - Revue de Pathologie somparée et d'Hygiène générale. - 5/20 sөpt. 1927.

(4) Mare Fouassier. - L'expertise des laits malpropres. - Annales des Falsifcations et des Fraudes, 1927, 20, 577.

(5) G.R. GAGE. - Studies of the life history of Ustilago avenae Jensen and Ustilago Levis Magn. - Cornell University, Agricultural Experiment Station. Mémoire 109. - Juillet 1927.

(6) Ch. Poncher. - Conférences faites à diverses Sociétés Vétérinaires de Franee, Rapport au Congrès Vétérinaire International de Londres, 1914, etc...

\section{BIBLIOGRAPHIE ANALYTIQUE}

\section{LES LIVRES}

E. Vallaud. - L'élevage dans la région de Saint-Benoit-duSault (Indre). Thèse Doct. Vét., Paris, 1928. Imprimerie Nouvelle, 7 , rue de la Regratterie, Poitiers, 1928. Une brochure de 72 pages.

Après des considérations sur l'aspect général du département de l'Indre et de la région de ce pays, $V$. nous parle des races bovines qui s'y trouvent, des anciennes races comme la Parthenaise, la Marchoise et la race Limousine qui tend actuellement à prédominer. faite.

C'est une étude zootechnique limitśe à un point de la France, mais très bien

\section{A. Delorme. - De l'acclimatation du bétail européen au Maroc.} Thèse Doct. Vét., Lyon, 1928. Une brochure de 68 pages, L'imprimerie française, Casablanca, 1928.

Il s'agit là d'une étude de zootechnie et de pathologie dans laquelle l'auteur, qui est rattaché au Service vétérinaire du Maroc, a rassemblé des documents très intéressants pour ceux qui voudraient aller faire de l'élevage dans ce pays. Il appelle 